\title{
Infiltrating Intramuscular Lipoma
}

National Cancer Institute

\section{Source}

National Cancer Institute. Infiltrating Intramuscular Lipoma. NCI Thesaurus. Code

C121131.

An infiltrating lipoma that affects skeletal muscle. It has a higher rate of local recurrence. 